\title{
Pituitary apoplexy associated with acute COVID-19 infection and pregnancy
}

\author{
Julie L. Chan ${ }^{1} \cdot$ Kimberly D. Gregory ${ }^{2} \cdot$ Sarah S. Smithson ${ }^{2} \cdot$ Mariam Naqvi $^{2} \cdot$ Adam N. Mamelak $^{1}$ (I)
}

Accepted: 29 August 2020 / Published online: 11 September 2020

๑) Springer Science+Business Media, LLC, part of Springer Nature 2020

\begin{abstract}
Purpose We report a case of a pregnant female presenting with pituitary apoplexy and simultaneous SARS-CoV-2 infection with a focus on management decisions.

Clinical history A 28-year-old G5P1 38w 1d female presented with 4 days of blurry vision, left dilated pupil, and headache. She tested positive for SARS-CoV-2 on routine nasal swab testing but denied cough or fever. Endocrine testing demonstrated an elevated serum prolactin level, and central hypothyroidism. MRI showed a cystic-solid lesion with a fluid level in the pituitary fossa and expansion of the sella consistent with pituitary apoplexy. Her visual symptoms improved with corticosteroid administration and surgery was delayed to two weeks after her initial COVID-19 infection and to allow for safe delivery of the child. A vaginal delivery under epidural anesthetic occurred at 39 weeks. Two days later, transsphenoidal resection of the mass was performed under strict COVID-19 precautions including use of Powered Air Purifying Respirators (PAPRs) and limited OR personnel given high risk of infection during endonasal procedures. Pathology demonstrated a liquefied hemorrhagic mass suggestive of pituitary apoplexy. She made a full recovery and was discharged home two days after surgery. Conclusion Here we demonstrate the first known case of successful elective induction of vaginal delivery and transsphenoidal intervention in a near full term gravid patient presenting with pituitary apoplexy and acute SARS-CoV-2 infection. Further reports may help determine if there is a causal relationship or if these events are unrelated. Close adherence to guidelines for caregivers can greatly reduce risk of infection.
\end{abstract}

Keywords Pituitary apoplexy $\cdot$ COVID- $19 \cdot$ Coronavirus $\cdot$ SARS-CoV-2 $\cdot$ Pregnancy

\section{Introduction}

Pituitary apoplexy (PA) is defined as the sudden hemorrhagic infarction of a pre-existing pituitary tumor, or in rare cases the gland itself. Typical symptoms of apoplexy include sudden onset of headache, visual loss, diplopia, nausea, fatigue, and hormonal deficiencies. The management of pituitary apoplexy is still somewhat debated. Patients with rapidly expanding mass lesions and severe or progressive visual loss or altered level of consciousness are typically operated on urgently to decompress the mass. Primary medical management often includes the use of corticosteroids

Adam N. Mamelak

Adam.Mamelak@cshs.org

1 Department of Neurosurgery, Cedars-Sinai Medical Center, Los Angeles, CA, USA

2 Cedars-Sinai Medical Center, Maternal Fetal Medicine, Los Angeles, CA, USA both to reduce edema of the ocular motor and optic nerves as well as to prevent symptoms of adrenal insufficiency due to loss of adrenocorticotrophic hormone (ACTH) secretion by the compressed or infarcted gland. PA is relatively rare, with an incidence of about $2-7 \%$ of pituitary adenomas [1]. It has been associated with a variety of co-morbidities including hypertension, diabetes, and anti-coagulation therapy [2, 3]. Apoplexy has been associated with pregnancy in several series, although the relationship between these two occurrences is unclear [4]. Theories underlying this connection include hypercoagulable state and hormonal influences due to pregnancy [5]. This exceedingly rare event has been reported less than fifty times in the gravid patient with a handful of reports $[4,6,7]$.

We are currently living through a pandemic of SARSCoV-2 (COVID-19) viral infection. While COVID-19 main symptoms include respiratory distress, headache, dry cough, and cardiac failure, a surprisingly large number of other symptoms have been identified [8-10]. To fully capture all 
potential related symptoms or diseases, we present a case of a near full-term pregnant female who presented with pituitary apoplexy in the setting of acute COVID-19 infection.

\section{Case history}

A 28-year-old gravida 5 para 1 female at 38 weeks 1-day gestational age pregnancy noted onset of blurriness in her left eye vision approximately 4 days prior to presentation to the hospital. She presented to urgent care where she denied diplopia but did endorse mild headache, sensation of decreased visual acuity in the left eye, and left ear pain. She was treated with oral amoxicillin for presumptive otitis media and sent home. Three days later she then re-presented with a new dilated left pupil and diminished visual acuity in that same eye. She had relatively minimal headache at this point. Neurological exam revealed anisocoria with the left pupil $5 \mathrm{~mm}$ in diameter and minimally reactive, and the right pupil $3 \mathrm{~mm}$ in diameter and reactive to light. The extraocular movements were intact with no diplopia appreciated. No formal visual fields were obtained, but on examination, visual fields were full to confrontation with only slight blurring of vision in her left eye. She underwent a head CT scan demonstrating a hemorrhagic mass in the region of the sella turcica, with an enlarged sella suggesting a previously undetected tumor. She was then transferred to our hospital for higher level of care where a routine COVID-19 screening questionnaire highlighted 1 week of ear pain, body aches, chills, and rhinorrhea. She also endorsed contact with her husband who was confirmed COVID-19 positive. Based on these screening criteria, she was placed on strict COVID-19 precautions with appropriate isolation measures and subsequent polymerase chain reaction (PCR) testing determined she was positive for SARS-CoV-2. Endocrine work-up demonstrated a low TSH of $0.28 \mathrm{mIU} / \mathrm{L}$ and a serum prolactin of $148.7 \mathrm{ng} / \mathrm{mL}$. Random cortisol was within normal limits, although she did receive a single dose of dexamethasone $10 \mathrm{mg}$ at the outside hospital. FSH and LH were $<0.1$ and $4.6 \mathrm{mIU} / \mathrm{mL}$ suggesting hypogonadism (Table 1). There was no evidence of diabetes insipidus. She was started on dexamethasone $4 \mathrm{mg}$ twice daily and within $12 \mathrm{~h}$ the left pupil decreased in size to $4 \mathrm{~mm}$ and was more reactive to light, with no other changes in examination. She reported her left eye visual acuity was subjectively better with decreased blurring. She denied any other neurological symptoms and her headache resolved.

As the patient was clinically stable on dexamethasone with an improving visual exam, surgery was deferred. MRI scan of the pituitary performed with and without contrast demonstrated a $2.2 \mathrm{~cm} \times 2.5 \mathrm{~cm} \times 2.0 \mathrm{~cm}$ cystic solid lesion with an expanded sella turcica (Fig. 1), suggesting a previously undetected pituitary tumor. There was a fluid-fluid
Table 1 Preoperative (1 week after symptom onset) and postoperative (2 months) hormone levels

\begin{tabular}{llll}
\hline Hormone & Reference value (range) & Preop & Postop \\
\hline Prolactin & $5.2-26.5 \mathrm{ng} / \mathrm{mL}$ & 148.7 & 33.8 \\
Cortisol & $3.7-19.4(\mathrm{AM}) \mu \mathrm{g} / \mathrm{dL}$ & $3.9^{\mathrm{a}}$ & 12.4 \\
ACTH & $6-50 \mathrm{pg} / \mathrm{mL}$ & $<5$ & 16 \\
TSH & $0.39-4.60 \mathrm{mIU} / \mathrm{L}$ & 0.28 & $<0.01^{\mathrm{a}}$ \\
T4 (free) & $0.70-1.48 \mathrm{ng} / \mathrm{dL}$ & 0.47 & $1.16^{\mathrm{b}}$ \\
FSH & $2.6-8.1 \mathrm{mIU} / \mathrm{mL}$ & $<0.1$ & 2.6 \\
LH & $24-105 \mathrm{mIU} / \mathrm{mL}$ & 4.6 & 3.7 \\
IGH-1 & $63-373 \mathrm{ng} / \mathrm{mL}$ & 103 & 143 \\
\hline
\end{tabular}

${ }^{\text {a }}$ Taking dexamethasone $8 \mathrm{mg} /$ day

${ }^{\mathrm{b}}$ Taking levothyroxine $100 \mathrm{mcg} / \mathrm{day}$

level consistent with acute hemorrhage and necrotic appearing tissue more posterior. The normal gland was compressed to the right with the optic chiasm slightly bowed. There was also extension into the left medial superior aspect of the cavernous sinus.

She was also evaluated by the maternal fetal medicine team who identified a healthy 38 -week pregnancy. Since the patient was clinically stable, after consultation among the maternal fetal medicine and neurosurgical services, it was decided the patient would undergo vaginal delivery under epidural anesthesia to prevent pushing, prior to the transsphenoidal surgery to remove the mass. She underwent induction on hospital day 5 at 38 weeks 6 days, with plan for delivery the next day at 39 weeks, and anticipation of surgery on postpartum day 2. During the delivery process, she received a planned epidural anesthetic and progressed to full cervical dilation without incident. She then underwent elective forceps assisted vaginal delivery to shorten the second stage and minimize any maternal pushing. Delivery was uncomplicated with the birth of a viable male infant weighing $3495 \mathrm{~g}$ with Apgar scores of 9 and 9 at one and $5 \mathrm{~min}$, respectively. Postpartum, the patient opted for formula feeding as this was also her preference with her prior child. Neonatal COVID-19 testing performed on day of life 2 was negative for coronavirus.

Two days after delivery she underwent endoscopic transsphenoidal surgery for removal of the mass. The primary impetus for decompression of the mass was the possibility for hormonal recovery and prevention of further hormonal deficit in this otherwise young healthy patient. A repeat nasopharyngeal PCR COVID-19 test prior to surgery was again noted to be positive. At surgery, high level COVID-19 precautions were carried out by all operating room personnel including the use of Powered Air-Purifying Respirators (PAPR), multiple layers of personal protective equipment (PPE), intubation by a single anesthesiologist in the room followed by a 15 min delay before other team members could 

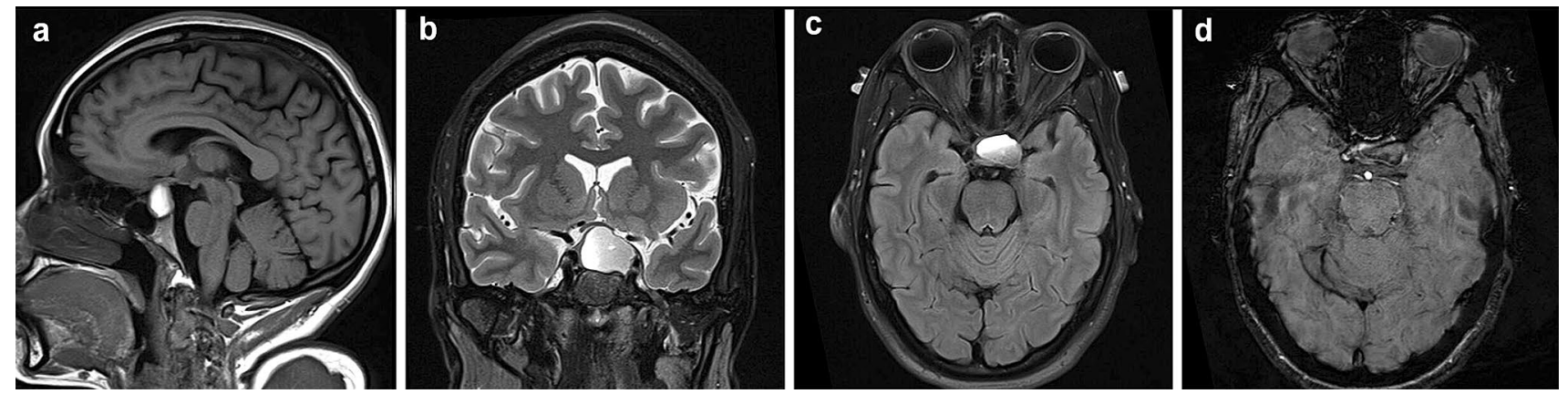

Fig. 1 MRI pituitary without contrast demonstrating $2.2 \mathrm{~cm} \times 2.5 \mathrm{~cm} \times 2.0 \mathrm{~cm}$ cystic solid lesion with an expanded sella turcica and hemorrhage on a sagittal T1, b coronal T2, $\mathbf{c}$ axial FLAIR, and $\mathbf{d}$ axial SWI sequences

enter the operating room, a minimal number of healthcare workers in the room during surgery (anesthesiologist, neurosurgeon, surgical scrub technician, and nurse circulator) and a 15 min delay after extubation with only the anesthesiologist in the room. Surgery was carried out without complication. At surgery, a predominantly liquefied hemorrhagic mass was identified with necrotic tissue particularly along the left posterior aspect of the sella which was markedly expanded. The gland was compressed superiorly and to the right-hand side. Final pathology demonstrated necrotic tissue without any evidence of viable tumor. Pituitary adenoma with apoplexy remains the most likely diagnosis given the radiographic appearance as well as the delayed intervention which may lead to the presence of only necrotic tissue. Postoperatively the patient made an excellent recovery with complete reversal of her pupillary defect and visual disturbances. She was placed on hydrocortisone $10 \mathrm{mg}$ in the morning and $5 \mathrm{mg}$ in the evening. A TSH on postoperative day 2 was normal at $0.99 \mathrm{mIU} / \mathrm{L}$ with a slightly low free T4 of $0.62 \mathrm{ng} /$ $\mathrm{dL}$. She was discharged home on postoperative day 2 with no evidence of hyponatremia or diabetes insipidus. Follow up at two months post-op demonstrated central hypothyroidism, and hypogonadism. She remained on levothyroxine 100 mcg daily. She continued hydrocortisone $10 / 5 \mathrm{mg}$ with normal serum cortisol levels but had not yet undergone cortisol stimulation testing to determine if secondary adrenal insufficiency persisted. (Table 1).

\section{Discussion}

Pituitary apoplexy is a relatively rare occurrence representing a clinical syndrome of expansion and hemorrhagic infarction of a pituitary adenoma which often results in acute compression of the pituitary gland and related loss of pituitary regulated hormonal function [3]. The exact definition of pituitary apoplexy remains somewhat debated with some focusing primarily on the radiological findings of hemorrhage and infarction $[11,12]$ others pointing out the need to correlate with clinical symptomatology and laboratory derangements [13, 14]. In most patients, the finding of any evidence of hemorrhage within the pituitary gland is in itself unlikely to meet the classification for apoplexy as hemorrhage within an incidentally discovered or even symptomatic pituitary tumor is relatively common occurring approximately $14 \%$ of cases but is not typically associated with clinical symptomatic apoplexy [11].

Apoplexy associated with a pregnancy is reported throughout the medical literature although the actual number of cases reported remains relatively small, with approximately 45 cases [4, 6, 7]. A review of this literature demonstrates that the majority occur in the second or third trimester, particularly the third trimester and the management of the apoplexy had relatively little impact on the delivery of the child or healthy completion of pregnancy. It is unclear what is the underlying pathophysiology of apoplexy during pregnancy or whether these are coincidental events. It is noted during pregnancy that the normal pituitary gland will enlarge up from 30 to $45 \%$ presumably due to increased estrogen causing lactotroph hyperplasia and increase in pituitary blood flow [5]. This enlargement of the gland however does not clearly lead to an apoplectic event as in the case of macroadenomas which have long expanded the sella prior to any of the events of pregnancy. Thus, it is not entirely clear whether the pregnancy itself is a contributing factor. Considering the relative frequency of pituitary adenomas and the common place nature of pregnancy it would seem this is more of a coincidental relationship rather than a causal one.

We report the first case of a COVID-19 positive patient in her third trimester presenting with pituitary apoplexy. COVID-19 is a new viral disease and we are just beginning to learn about its many manifestations. It is unclear whether this the COVID-19 infection was a contributing factor in the apoplectic event, or if these events were coincidental and unrelated. Hemorrhagic complications of COVID-19 infection are beginning to be reported with several reports indicating increased risk for cerebrovascular hemorrhage along with cardiac and kidney related infarction [15-18]. 
It is possible given the relatively fragile nature of the vasculature in the pituitary region that a coronavirus related infection could contribute to acute hemorrhagic infarction of the gland and related apoplexy although at this point this remains entirely speculative.

From a management point of view the approach taken was to manage each of the individual medical problems with the patient as separate and unrelated. Due to the large size of the mass with associated visual changes, surgical decompression seemed appropriate. Recent recommendations by Fleseriu et al. suggest categorizing COVID-19 positive patients in need of pituitary surgery as either emergent, urgent, or elective in regard to operative intervention timing. In our case, the patient fell somewhere in between urgent and elective as she appeared to have pituitary apoplexy but was stable on steroids without neurologic deterioration [19]. Non-operative conservative management was highly considered and may have in fact been the preferred option in a stable patient with pituitary apoplexy during this coronavirus pandemic given the concern for spread of the disease via aerosolization of the virus. However, there are a number of studies and reviews which suggest that operative intervention within a week for pituitary apoplexy may be beneficial over conservative management in the setting of severe progressive visual loss, altered consciousness, or even in cases of cranial neuropathies as was in this case [20-23]. Further, given her protracted clinical course, intent to decrease steroid utilization in the postpartum state, and concern for reliable follow up, the decision was made to pursue operative intervention on an elective basis. The decision was also made to electively deliver the child without pushing to avoid any further pressure on the optic nerves due to mass-effect from the apoplectic lesion. Further, elective induction of vaginal delivery removed the need for additional anesthetic or operative precautions or consideration during surgery. Regarding the patient's COVID-19 positive status, vaginal delivery is low risk to the fetus/newborn in patients with mild-moderate apoplexy symptoms, as was the case with our patient [24].

Surgical removal of the pituitary lesion was straightforward. However, due to the patient's COVID-19 status, complete respiratory and contact related precautions were carried out. Of note there is recent literature indicating that patients with COVID-19 represent a very high-risk population first disseminating virus to healthcare workers during transsphenoidal surgery presumably due to aerosolization of viral particles [25]. As described in recent recommendations by the Pituitary Society, it is critical that healthcare workers performing any form of endonasal surgery carry out strict adherence to related protective strategies including no direct patient contact without the use of PPE and PAPR devices, appropriate donning and doffing techniques, and careful showering after completion of the surgical procedure [19]. These were all carried out according to recommended guidelines with no postoperative evidence of infection in any of the clinical staff involved in the care of this patient.

As more information is gained about the COVID-19 virus other case reports may be able to shed further insight. At present this represents the first documented case of pituitary apoplexy in a pregnant woman with COVID-19 and may serve as a useful guideline for further case reports or series within the medical literature.

Author contributions All authors made substantial contributions to the conception and design of the work as well as drafted, revised, and approved the version to be published. All authors agree to be accountable for all aspects of the work related to accuracy and integrity.

Funding This research did not receive any specific grant from funding agencies in the public, commercial, or not-for-profit sectors.

\section{Compliance with ethical standards}

Conflict of interest The authors have no conflicts of interests or competing interests to disclose.

\section{References}

1. Rajasekaran $\mathrm{S}$ et al (2011) UK guidelines for the management of pituitary apoplexy. Clin Endocrinol (Oxf) 74:9-20

2. Briet C, Salenave S, Bonneville J, Laws ER, Chanson P (2015) Pituitary Apoplexy. Endocr Rev 36:622-645

3. Glezer A, Bronstein MD (2015) Pituitary apoplexy: pathophysiology, diagnosis and management. Arch Endocrinol Metab 59:259-264

4. Piantanida E et al (2014) Pituitary apoplexy during pregnancy: a rare, but dangerous headache. J Endocrinol Invest 37:789-797

5. Karaca Z, Tanriverdi F, Unluhizarci K, Kelestimur F (2010) Pregnancy and pituitary disorders. Eur J Endocrinol 162:453-475

6. Jemel M et al (2019) Gestational pituitary apoplexy: Case series and review of the literature. J Gynecol Obstet Hum Reprod 48:873-881

7. Grandaison S, Weber F, Bédard MJ, Mahone M, Godbout A (2015) Pituitary apoplexy in pregnancy: a case series and literature review. Obstet Med 8:177-183

8. Jin $X$ et al (2020) Epidemiological, clinical and virological characteristics of 74 cases of coronavirus-infected disease 2019 (COVID-19) with gastrointestinal symptoms. Gut 69:1002-1009

9. Chen $\mathrm{N}$ et al (2020) Epidemiological and clinical characteristics of 99 cases of 2019 novel coronavirus pneumonia in Wuhan, China: a descriptive study. Lancet 395:507-513

10. Yang X et al (2020) Clinical course and outcomes of critically ill patients with SARS-CoV-2 pneumonia in Wuhan, China: a singlecentered, retrospective, observational study. Lancet Respir Med 8:475-481

11. Kinoshita Y et al (2014) Impact of subclinical haemorrhage on the pituitary gland in patients with pituitary adenomas. Clin Endocrinol (Oxf) 80:720-725

12. Boellis A, di Napoli A, Romano A, Bozzao A (2014) Pituitary apoplexy: an update on clinical and imaging features. Insights Imaging 5:753-762

13. Barkhoudarian G, Kelly DF (2019) Pituitary apoplexy. Neurosurg Clin North Am 30:457-463 
14. Semple PL, Jane JA, Lopes MBS, Laws ER (2008) Pituitary apoplexy: correlation between magnetic resonance imaging and histopathological results. J Neurosurg 108:909-915

15. Lodigiani $\mathrm{C}$ et al (2020) Venous and arterial thromboembolic complications in COVID-19 patients admitted to an academic hospital in Milan. Italy Thromb Res 191:9-14

16. Klok FA et al (2020) Incidence of thrombotic complications in critically ill ICU patients with COVID-19. Thromb Res 191:145-147

17. Sharifi-Razavi A, Karimi N, Rouhani N (2020) COVID-19 and intracerebral haemorrhage: causative or coincidental? New Microbes New Infect 35:100669

18. Poyiadji N et al (2020) COVID-19-associated Acute Hemorrhagic Necrotizing Encephalopathy: Imaging Features. Radiology 296:E119-E120

19. Fleseriu M et al (2020) Pituitary society guidance: pituitary disease management and patient care recommendations during the COVID-19 pandemic - an international perspective. Pituitary 23:327-337

20. Kim SH, Lee KC, Kim SH (2007) Cranial nerve palsies accompanying pituitary tumour. J Clin Neurosci 14:1158-1162
21. Almeida JP et al (2019) Pituitary Apoplexy: Results of Surgical and Conservative Management Clinical Series and Review of the Literature. World Neurosurg 130:e988-e999

22. Bi WL, Dunn IF, Laws ER (2015) Pituitary apoplexy. Endocrine 48:69-75

23. Seo Y et al (2018) The Outcomes of Pituitary Apoplexy with Conservative Treatment: Experiences at a Single Institution. World Neurosurg 115:e703-e710

24. Ferrazzi E et al (2020) Vaginal delivery in SARS-CoV-2-infected pregnant women in Northern Italy: a retrospective analysis. BJOG An Int J Obstet Gynaecol 127:1116-1121

25. Patel ZM et al (2020) Letter: Precautions for endoscopic transnasal skull base surgery during the COVID-19 pandemic. Neurosurgery 87:E66-E67

Publisher's Note Springer Nature remains neutral with regard to jurisdictional claims in published maps and institutional affiliations. 